\title{
First mirror tests for ITER: influence of material choice on the erosion/deposition mechanisms affecting optical reflectivity.
}

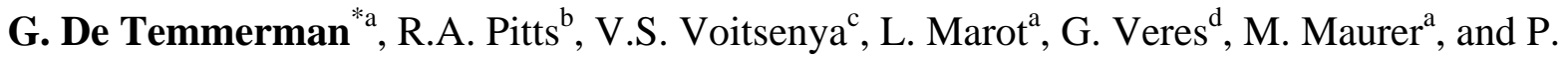 \\ Oelhafen $^{\mathrm{a}}$ \\ ${ }^{a}$ Institute of Physics, University of Basel, CH-4056 Basel, Switzerland \\ ${ }^{b}$ Ecole Polytechnique Fédérale de Lausanne (EPFL),Centre de Recherches en Physique des Plasmas, \\ Association EURATOM, Confédération Suisse, 1015 Lausanne, Switzerland \\ ${ }^{c}$ Institute of Plasma Physics, NSC KIPT, Akademichna St. 1, 61108 Kharkov, Ukraine \\ ${ }^{d}$ KFKI Research Institute for Particle \& Nuclear Physics, EURATOM Association POB 49, H-1525 \\ Budapest, Hungary
}

\begin{abstract}
:
Almost all optical diagnostics systems on ITER will be based on in-vessel metallic first mirrors. The possible deterioration of their surface reflectivity as a result of erosion by charge-exchange neutrals and re-deposition of material eroded from the plasma-facing components represents a serious concern for the reliability of spectroscopic and laser signals. A concerted effort within the tokamak community has been initiated to characterize these effects and seek mitigation methods. To date, the different damaging effects (erosion and deposition) have always been considered independently, neglecting any role that may be played by the substrate. In this contribution, we attempt to assess the influence of substrate material using different approaches: laboratory simulation experiments, mirror exposures in the divertor region of the TCV tokamak and numerical simulations using the Monte-Carlo code TRIDYN. Our results have potentially important implications for the choice of ITER first-mirror materials.
\end{abstract}

PACS: 52.40.Hf, 52.55.Fa, 52.80.Hc, 78-20.ci

JNM keywords: Optical properties, Plasma-material interactions, Surface processes, Carbon.

PSI-16 keywords: Material properties, Erosion/deposition, Reflectivity, TCV, ITER 
*Corresponding author address: Institute of Physics, Klingelbergstrasse 82, CH-4056 Basel, Switzerland

*Corresponding author e-mail: gregory.detemmerman@unibas.ch

Presenting author: Gregory De Temmerman

\section{Introduction:}

Plasma diagnostic systems will be critical elements in future fusion reactors such as ITER (International Thermonuclear Experimental Reactor), both for the usual measurement of key plasma parameters but also for the real time control that will be required in a long pulse, high power device. In today's tokamaks, the plasma is viewed through optical windows or fibre optics. However, the high level of radiation expected in ITER will render this conventional approach impossible. In-vessel metallic mirrors are therefore planned for use with the majority of optical diagnostics [1]. The possible deterioration of their reflectivity as a result of erosion by charge-exchange (CX) neutrals and re-deposition of material eroded from plasmafacing components represents a serious concern for the reliability and long term usefulness of spectroscopic systems [2].

The effects of these different damaging mechanisms have been previously studied in various laboratory experiments [2-4]. Dedicated experimental programmes have also recently been initiated in various tokamaks to further address this issue [5-8]. In these experiments, high Z materials in the form of single crystals, such as molybdenum, have demonstrated their ability to withstand erosion conditions with a sufficient lifetime, but to date, deposition and erosion mechanisms have always been considered separately. However, as shown in [9], in the case of the simultaneous bombardment of a substrate by deuterium and carbon, the transition between erosion and deposition is a function of the contaminant flux, the plasma temperature and the sputtering yield of the material (i.e. of the material itself). Differences in the rate of carbon film growth have also been observed in experiments on PISCES-B with carbon contaminated hydrogen plasmas [10]. These observations are of obvious importance for first mirror 
materials in ITER. Dedicated efforts, summarised here, have therefore been initiated to compare the erosion/deposition patterns observed on different materials under similar conditions, using laboratory simulation experiments, mirror exposures in the TCV tokamak and numerical simulations.

\section{Laboratory experiments}

\section{a. Experimental}

Mirrors made from stainless steel (SS) and polycrystalline copper have been exposed to a deuterium glow discharge with controlled partial pressure of methane in the gas mixture. Experiments are performed in a high vacuum plasma chamber pumped down to a base pressure of about $10^{-5} \mathrm{~Pa}$ using a conventional pumping system. Monitoring of the neutral pressure during the plasma exposure is achieved using a mass spectrometer located in an auxiliary chamber connected to the main plasma chamber through a $100 \mu \mathrm{m}$ diameter pinhole. This measurement provides $f_{\mathrm{CH}_{4}}$, the methane content in the gas mixture. The mirrors are mounted on a water-cooled sample holder and a negative bias of -200 V applied on the mirror controls the impinging ion energy. Ion fluxes are determined by measuring the current passing through the sample, neglecting secondary electron emission. Reflectivity data for s-polarized light are measured using a real time laser reflectometer $[11](\lambda=532 \mathrm{~nm})$ with incidence angle $52^{\circ}$ during the plasma exposure itself. Plasma parameters $\left(n_{e} \cong 10^{15} \mathrm{~m}^{-3}\right.$ and $\left.T_{e}=13 \mathrm{eV}\right)$ were measured by means of a Langmuir probe.

Scanning Electron Microscopy (SEM) is used to study the surface morphology and Energy Dispersive X-Ray analysis (EDX) to check the surface chemical composition. Weight measurements of the mirrors before and after plasma exposure together with stylus profilometer measurements allow the eroded/deposited depth to be determined.

The mirror samples were prepared in IPP Kharkov, Ukraine. All samples were cut using arc cutting in kerosene, washed by acetone and exposed for 20 minutes to low energy ions 
(50 eV) from a hydrogen ECR plasma. Since copper is very sensitive to oxidation in air, the samples were cleaned again for 5 minutes in a deuterium plasma (ion energy $200 \mathrm{eV}$ ) before the experiment.

\section{b. Results}

Three different deuterium/methane mixtures were used: $f_{\mathrm{CH}_{4}}=0, f_{\mathrm{CH}_{4}}=1.8 \%$ and $f_{\mathrm{CH}_{4}}=3.5 \%$. Figure 1 shows the evolution of the reflectivity (R) of the mirrors as a function of the ion fluence during plasma exposure; the values have been normalized to the initial value for ease of comparison. Results obtained for the two materials are quite different. In the case of stainless steel (fig. 1.a), for $f_{\mathrm{CH}_{4}}=0$ and $1.8 \%$, the evolution of the reflectivity with ion fluence is linear, showing a final value of 0.96 after a fluence of $8 \cdot 10^{19} \mathrm{~cm}^{-2}$. For higher methane content in the gas mixture, constructive and destructive interferences typical of the growth of an amorphous hydrogenated carbon film (a-C:H) are observed. For copper (fig. 1.b), the degradation rate of the reflectivity is directly related to the methane content in the gas mixture: the higher the methane content the faster the reflectivity decreases. At $f_{\mathrm{CH}_{4}}=3.5 \%$, the reflectivity falls essentially to zero for a fluence of $1 \cdot 10^{19} \mathrm{~cm}^{-2}$.

Investigation of the surface morphology by SEM provides some clues regarding the damaging effect of the plasma exposure for the two materials. For $f_{\mathrm{CH}_{4}}=0$ and $1.8 \%$, the stainless steel mirror surface remains undamaged (fig. 2.a), with no sign of the stepped-structure found, for example in [12] for polycrystalline samples exposed to similar deuterium ion fluence. The roughness remains very low $\left(\mathrm{R}_{\mathrm{a}}=6 \mathrm{~nm}\right.$ compared to an initial value of $\left.5 \mathrm{~nm}\right)$ for both cases, consistent with the slight change observed for the specular reflectivity in figure 1 . It should be noted that EDX analysis finds no trace of carbon on samples exposed to $f_{\mathrm{CH}_{4}}=0$ and $1.8 \%$. For copper, the evolution of the surface morphology with $f_{\mathrm{CH}_{4}}$ is very different. For $f_{C_{4}}=0 \%$, neither visible damage of the surface (fig. 2.b) nor any increase in the surface 
roughness is found ( $R_{a}=7 \mathrm{~nm}$ compared to an initial value of $\left.6 \mathrm{~nm}\right)$. However, when the methane content is increased, the different crystallographic grains start to become visible (fig. 2.c); the effect being more pronounced for $f_{\mathrm{CH}_{4}}=3.5 \%$ (fig. 2.d). The resulting strong surface roughness increase explains the degradation of the reflectivity described above.

Figure 3 summarises the eroded/deposited depth measured for the different mirrors, showing very different behaviour of the two substrates under similar conditions. From $f_{\mathrm{CH}_{4}}=0 \%$ to $f_{\mathrm{CH}_{4}}=1.8 \%$, the reduced erosion of the stainless steel mirror seems to indicate a "carbon protection" of the surface: the flux of carbon radicals arriving at the surface is insufficient to fully compensate the number of atoms removed by sputtering (thus preventing the transition from erosion to deposition), but high enough to partially protect the surface from plasma ion sputtering. For higher methane content, the surface becomes coated with an a-C:H film. In the case of copper there is a strong increase in the eroded depth from $f_{\mathrm{CH}_{4}}=0 \%$ to $f_{\mathrm{CH}_{4}}=1.8 \%$, with only a slight reduction of the sputtered depth at the highest methane content.

\section{Exposure of different mirror materials in the TCV tokamak}

\section{a. Experimental}

Mirror samples from a variety of different materials of interest to ITER (Mo, W, Si) are prepared at the University of Basel and installed in pairs on a specially designed manipulator [8] allowing sample insertion into the divertor floor region of the TCV tokamak (nearly $90 \%$ graphite first wall coverage). A separate pumping system allows the mirrors to be easily inserted and retrieved following exposure without requiring a vacuum vessel vent. Samples are recessed below the front surface of the divertor tiles to avoid direct plasma ion impact. The manipulator location places the samples underneath the far divertor SOL of standard TCV single null lower discharges. The large variety of magnetic equilibria studied on TCV and the absence of any shutter system protecting the samples means that most exposures are integrated across short campaign periods of 2-3 weeks, including regular helium glow 
discharge conditioning. To minimize the energy of the impinging He ions during the glow, the sample manipulator is electrically insulated from the rest of the torus placing the samples at floating potential during the discharge and avoiding the $\sim 400 \mathrm{~V}$ cathode fall present elsewhere on the first wall.

Before and after each sample exposure, optical measurements and surface analysis are performed at the University of Basel. Spectroscopic ellipsometry is used to determine the optical properties of the samples and the deposited layer thickness, whilst XPS and SEM provide information on the layer composition and morphology. As with the laboratory exposed samples, a stylus profilometer is used to confirm the estimation of the deposited thickness.

\section{b. Main results}

Table 1 summarises the different materials tested, the experimental conditions (recessment distance, number of tokamak shots, glow discharge conditioning time etc.) and estimation of the deposited thickness. Evidently, only very thin layers have been found on the different samples, especially when high-Z materials (Mo, W) have been exposed. No difference in the carbon layer thickness was found when Mo and W were exposed simultaneously. There appears to be no correlation between the deposited thickness and the recess distance below the divertor target tiles, though the significance of this result must be gauged against the large differences in terms of plasma configurations and conditions (ohmic, H-mode, high power, low density electron cyclotron heated discharges, limiter, divertor etc.) characterising the different sample exposure periods. The most striking result is obtained when molybdenum and silicon samples are simultaneously exposed. The deposition rate on the Si sample is found to be much higher than on Mo, a phenomenon observed in two separate experiments. Since the exposure in pairs ensures that the samples experience nearly identical conditions, the only parameter which can play a role is the substrate material itself. It would therefore appear that 
the deposition efficiency on different substrates is quite different: carbon deposition on a high-Z material like Mo being less favourable than on Si.

The composition of the deposited layer has been investigated by XPS and SIMS, and found to be mainly deuterium and carbon, as expected given the use of deuterium as main plasma fuel and the extensive graphite coverage of the TCV first wall. However, in the current manipulator design, there is no possibility to measure the particle fluxes and energies the samples are submitted to.

\section{Numerical simulations}

The TRIDYN Monte Carlo simulation code [13] has been used to try and shed some light on the different erosion/deposition patterns observed both in laboratory experiments and in TCV, but with emphasis on the laboratory tests using different methane concentrations. Simulation parameters were chosen to reproduce the sample exposures made during laboratory experiments, and were defined as follow:

- electronic temperature of $13 \mathrm{eV}$ and sheath potential of -200 V (using the Langmuir probe measured $\mathrm{T}_{\mathrm{e}}$ and with the $-200 \mathrm{~V}$ corresponding to the bias potential applied to the samples),

- $\mathrm{D}^{+}$and $\mathrm{C}^{3+}$ as impinging ions (according to ionisation-recombination balance calculation $\mathrm{C}^{3+}$ is the majority carbon species in the plasma),

- maxwellian ion energy distribution,

- isotropic angular distribution (which is transformed to a more normal incidence by the sheath potential).

Table 2 shows the ion reflection coefficients determined by the code for the different substrates. The reflection coefficient of $\mathrm{C}$ is found to be higher on Mo than on Si. Carbon has less chance of being deposited on a Mo surface, and the particles reflected back off the substrate could also sputter the deposited layer reducing therefore the deposition rate. This 
would be in agreement with the experimental findings. For $\mathrm{Cu}$ and SS however, the reflection coefficients found for both materials are very close (as well as the eroded/deposited depth predicted by the code) which is not in agreement with the large discrepancies described above. The differences observed cannot be explained by physical effects only. However, chemical effects such as carbide formation are not accounted in the code. Since stainless steel is mainly composed of carbide forming elements and copper does not have a stable carbide, the surface chemistry and its influence on the stickiness of carbon may be playing an important role.

\section{Summary and discussion}

The results presented here demonstrate that under similar exposure conditions, mirrors from different materials behave quite differently. Under simultaneous bombardment with deuterium and carbon ions, a copper mirror is damaged by physical sputtering from the plasma ions whilst a stainless steel mirror becomes coated with an a-C:H film. For both substrates the mechanism affecting the optical reflectivity is therefore different: sputtering of the copper surface increases the surface roughness and thus decreases the reflectivity, whilst the reflectivity of the SS mirror is modified by the absorption of light in the deposited layer. The different deposition rates measured on Si and Mo after exposure in TCV under similar conditions confirm that the erosion/deposition pattern is substrate dependent.

The reason for these differences is not yet fully understood and further experiments are required to assess these effects. It is clear, however, that in choosing diagnostic first mirror materials in ITER, both the resistance of a material to sputtering and the efficiency of deposition (eg. C, Be, W) must be considered with equal importance.

\section{Acknowledgement:}

The financial support of the Federal Office for Education and Science and of the Federal Office of Energy is gratefully acknowledged. 


\section{References:}

[1] A. Costley et al. Fusion. Eng. and Des. 55 (2001) 331.

[2] V.S. Voitsenya et al, Rev. Sci. Instrum. 72 (2001) 475.

[3] V.S. Voitsenya et al, J. Nucl. Mat. 290-293 (2001) 336.

[4] T. Sugie et al, J. Nucl. Mat. 329-333 (2004) 1481.

[5] P. Wienhold et al, J. Nucl. Mat. 337-339 (2005) 1116.

[6] G. De Temmerman et al, Proc. of the 32nd EPS conference on Plasma Physics, Tarragona, Spain, June 2005.

[7] M. Lipa et al, Fus. Eng. and Des. 81 (2006) 221.

[8] M. Rubel et al., Rev. Sc. Inst., in press.

[9] D. Naujoks and W. Eckstein, J. Nucl. Mat. 230 (1996) 93.

[10] F. C.Sze et al, J. Nucl. Mater. 246 (1997) 165.

[11] A. Schüler et al, J. Appl. Phys. 87-9 (2000) 4285.

[12] A. Bardamid et al, Vacuum 58 (2000) 10-15.

[13] W. Möller et al, Comput. Phys. Commun. 51 (1988) 355.

\section{Figure captions:}

Figure 1: Real-time evolution of the specular reflectivity (R) of stainless steel (a) and copper (b) mirrors during laboratory plasma exposure. Typical exposure time of the sample is about 10 hrs.

Figure 2: SEM pictures of the surface morphology of the stainless steel mirror exposed to $f_{\mathrm{CH}_{4}}=1.8 \%$ (a), of the copper mirrors exposed to $f_{\mathrm{CH}_{4}}=0 \%$ (b); $1.8 \%$ (c) and $3.5 \%$ (d). Mean values of the roughness $\left(\mathrm{R}_{\mathrm{a}}\right)$ are indicated. 
Figure 3: Surface thickness changes of the different mirrors exposed in laboratory plasma experiments as determined from weight measurements and profilometry as a function of the methane content in the gas mixture, for equivalent normalized fluence.

Table 1: Experimental condition of the mirror exposures in TCV. $\mathrm{d}$ is the distance between the sample surface and the front surface of the graphite divertor tiles.

Table 2: Reflection coefficients for deuterium and carbon ions determined by numerical simulations with TRIDYN. 
Figure 1:
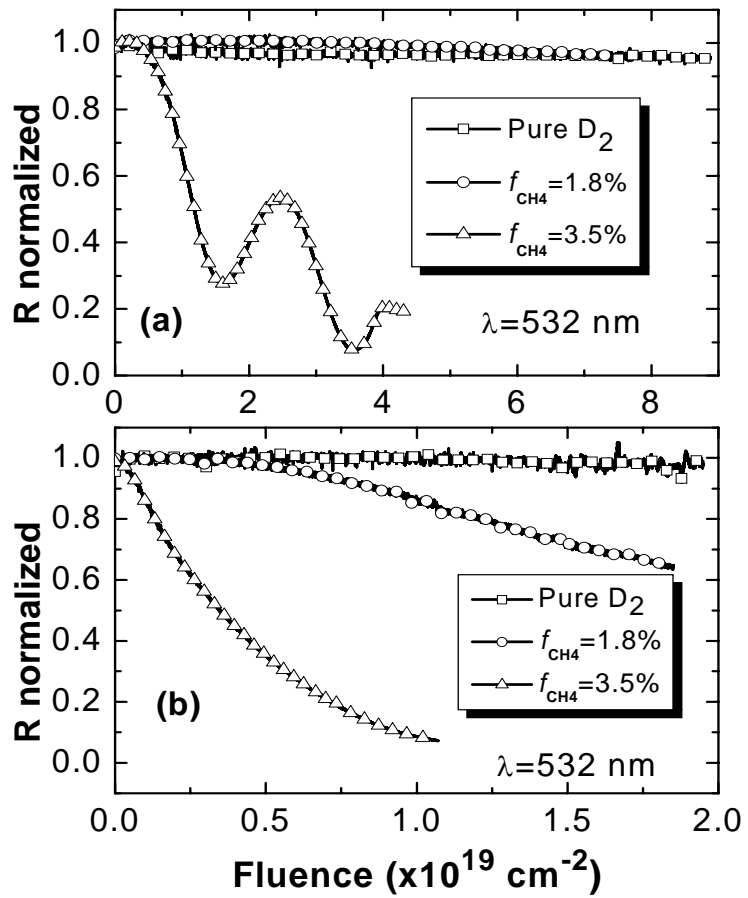
Figure 2:

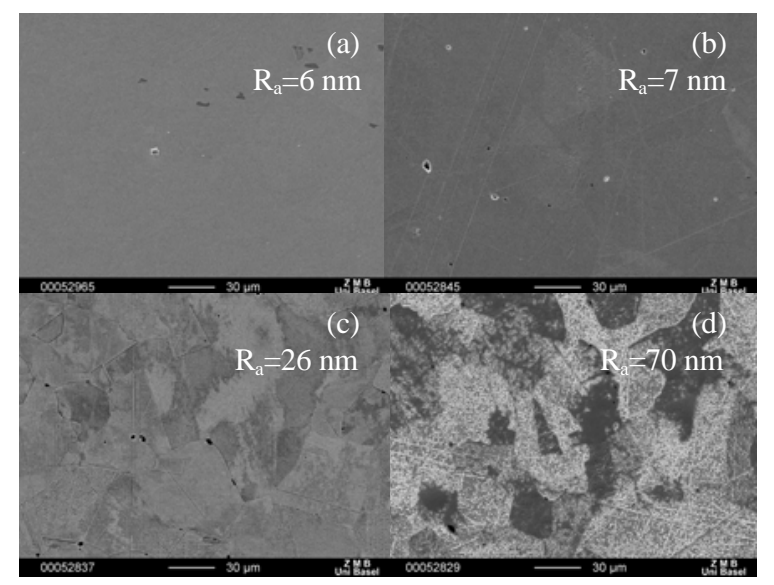


Figure 3:

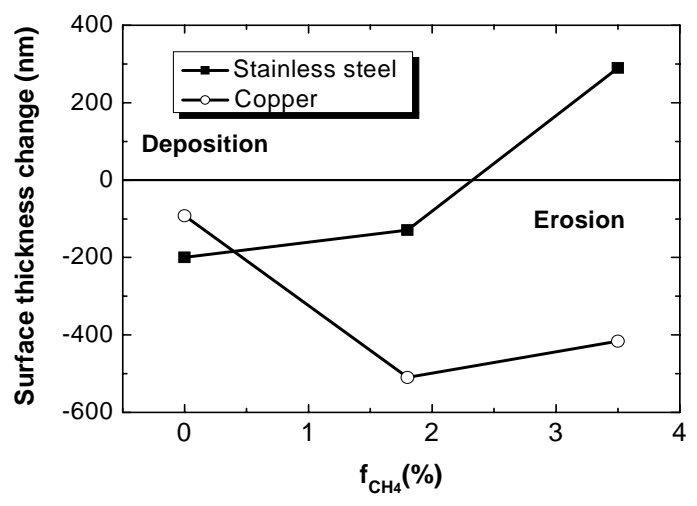


Table 1:

\begin{tabular}{|c|c|c|c|c|}
\hline Material & $\mathrm{d}(\mathrm{mm})$ & $\begin{array}{l}\mathrm{Nb} \text { of } \\
\text { shots }\end{array}$ & $\begin{array}{c}\text { Glow } \\
\text { discharge } \\
\text { (hrs) }\end{array}$ & $\begin{array}{l}\text { Deposited } \\
\text { thickness } \\
(\mathrm{nm})\end{array}$ \\
\hline $\begin{array}{l}\text { Mo } \\
\text { Mo }\end{array}$ & 15 & 323 & 33.44 & 4.7 \\
\hline $\begin{array}{c}\text { Mo } \\
\mathrm{W}\end{array}$ & 10 & 19 & 1.47 & 1 \\
\hline $\begin{array}{l}\text { Mo } \\
\mathrm{W}\end{array}$ & 50 & 214 & 21.54 & 0.9 \\
\hline $\begin{array}{c}\text { Mo } \\
\text { Si }\end{array}$ & 50 & 223 & 24.5 & $\begin{array}{c}1.3 \\
15.9\end{array}$ \\
\hline $\begin{array}{c}\text { Mo } \\
\text { Si }\end{array}$ & 50 & 820 & 90.5 & \begin{tabular}{|l|}
4 \\
24
\end{tabular} \\
\hline
\end{tabular}


Table 2:

\begin{tabular}{ccc}
\hline \multirow{2}{*}{ Material } & \multicolumn{2}{c}{ Ion } \\
\cline { 2 - 3 } & $\mathrm{D}$ & $\mathrm{C}$ \\
\hline $\mathrm{Cu}$ & 0.36 & 0.16 \\
\hline $\mathrm{Fe}$ & 0.32 & 0.13 \\
\hline $\mathrm{Mo}$ & 0.4 & 0.22 \\
\hline $\mathrm{Si}$ & 0.17 & 0.02 \\
\hline
\end{tabular}

